\title{
ELETROCENTROS E SISTEMA INTEGRADO DE AUTOMAÇÃO*
}

Marcelo Eduardo Rocha Gesualdi ${ }^{1}$

\section{Resumo}

A solução de eletrocentros aplicada a subestações elétricas dentro de uma planta industrial vem se consolidando no processo de mineração no Brasil em virtude de suas diversas vantagens de modularização, montagem e testes em fábrica, acelerando a instalação e energização das subestações em campo aonde os prazos de disponibilidade são sempre curtos para o início da operação da planta. Adicionalmente ao conceito de eletrocentros, a automação de subestações elétricas tem possibilitado através da utilização de redes de comunicação baseadas na norma IEC 61850 uma nova visão de integração dos diversos equipamentos, painéis elétricos e de automação que compõem as subestações dentro de uma plataforma integrada com a automação de processos da planta, disponibilizando um maior número de informações para o correto controle da subestação e gerenciamento de energia, bem como a redução significativa do cabeamento interno de controle e comando entre painéis facilitando ainda mais a modularização dos eletrocentros. Além das vantagens de montagem e comissionamento, o conjunto eletrocentro e sistema integrado de automação possibilita para a operação e a manutenção da planta consideráveis avanços através do gerenciamento remoto e integrado de informações, garantindo uma maior eficiência em seus respectivos processos.

Palavras-chave: Eletrocentro; Automação de subestações elétricas; IEC 61850.

\section{E-HOUSES AND AUTOMATION INTEGRATED SYSTEM}

\begin{abstract}
The E-House solution applied to electrical substations within an industrial plant has been consolidated in the mining process in Brazil because of its advantages of modularization, factory assembly and tests, speeding up the substation startup on the field where the availability deadlines are always short for the plant operation. In addition to the concept of E-House, electrical substation automation has enabled through the use of communication networks based on IEC 61850 a new integration vision of the various equipment, electrical and automation panels inside the substation within a single integrated architecture with process automation, providing a large amount of information to the correct substation control and power management, as well as significantly reducing the control wiring among panels further facilitating the E-House modularization. The complete solution of E-House and automation integrated system also allows the plant operation and maintenance considerable advances through remote and integrated information management, ensuring better efficiency in their process.
\end{abstract}

Keywords: E-House; Electrical substation automation; IEC 61850.

Engenheiro eletricista, gerente de engenharia - sistemas de automação e eletrificação, $A B B$, Osasco, SP, Brasil. 


\section{INTRODUÇÃO}

Eletrocentros são subestações elétricas transportáveis, pré-fabricadas e précomissionadas através do conceito de modularização em um ambiente de fábrica. Esse projeto modular baseado em estruturas metálicas proporciona uma alta flexibilidade para uma grande variedade de aplicações, ou seja, desde pequenas salas elétricas de um único módulo (Skids) a serem instaladas sobre máquinas móveis até grandes subestações elétricas de diversos módulos mecânicos para completar dimensões de até $800 \mathrm{~m}^{2}$. Os eletrocentros podem ser projetados seguindo diferentes especificações e requisitos das indústrias de processo e locais de instalação, sendo que para a indústria de mineração tem se verificado um aumento significativo desse tipo de solução em virtude das dificuldades de implantação em campo das tradicionais subestações elétricas de alvenaria que demandam um maior gerenciamento de recursos e materiais pelas longínquas distâncias de tais empreendimentos.

Equipamentos elétricos de média e baixa tensão típicos de uma subestação tais como painéis de distribuição, centros de controle de motores, inversores de frequência, transformadores e outros são instalados e testados em conjunto com o sistema de automação de processos e sistemas de utilidades que compõem o eletrocentro para envio ao local definitivo da planta. Dessa maneira, o trabalho de montagem da subestação pode ser executado independente das diversidades do campo como condições ambientais, climáticas e de segurança, além de atrasos e interferências em obra, uma vez que todo o trabalho é realizado em um ambiente controlável de fábrica, aonde todas as ferramentas, máquinas de movimentação, materiais de instalação e recursos humanos especializados para montagem e testes estão disponíveis.

A solução completa de eletrocentro reduz o período de montagem e comissionamento em campo em virtude dos eletrocentros serem entregues com todos os equipamentos pré-comissionados e pré-testados da fábrica, bem como evita praticamente grande parte do projeto e construção civil que se limitam somente a disponibilidade da fundação aonde os eletrocentros serão instalados.

Adicionalmente as facilidades de implantação em campo da solução de eletrocentros, a integração dos sistemas elétricos com o sistema de automação através de redes de comunicação baseadas na norma IEC 61850 e dispositivos eletrônicos inteligentes (IED - intelligent electronic device) proporciona diversas vantagens para operação e manutenção das subestações elétricas. A implementação de funções de controle de potência e seletividades lógicas associadas à grande quantidade de informações dos IED's disponibilizada via comunicação para o sistema de automação, permitem uma melhora no gerenciamento de energia e controle da planta como um todo, uma vez que todos as subestações podem ser interligadas em um único sistema de automação independente das distâncias entre elas.

Por outro lado, a manutenção da planta é beneficiada pela possibilidade de otimização dos ativos das subestações elétricas uma vez que o gerenciamento remoto e integrado das informações pode garantir uma maior eficiência na aplicação de um processo de manutenção preditiva e consequentemente aumentando a disponibilidade operacional e produtiva da planta. 


\subsection{Características Mecânicas dos Eletrocentros}

O eletrocentro possui um projeto modularizado para facilitar a montagem em fábrica e principalmente em campo. A modularização permite o transporte do conjunto eletrocentro em partes dentro dos dimensionais aceitáveis e então posicionamento e reconexão dos módulos em campo. O projeto estrutural mecânico do eletrocentro é fundamental para garantir a correta modularização do eletrocentro.

Para o projeto mecânico e contrução da estrutura do eletrocentro deve-se considerar sua rigidez mecânica, pois a sala deverá suportar a massa de sua estrutura somada com a massa dos componentes instalados, além de cargas adversas como a do vento e sísmicas quando aplicáveis. A rigidez da estrutura também deve ser considerada para os processos de movimentação como transporte da fábrica para o campo e içamentos para posicionamento dos módulos sobre as fundações finais. Para garantir a rigidez da estrutura, todo o projeto é concebido e suas cargas simuladas em uma plataforma de software de engenharia específica para análise estrutural através do modelamento por elementos finitos.
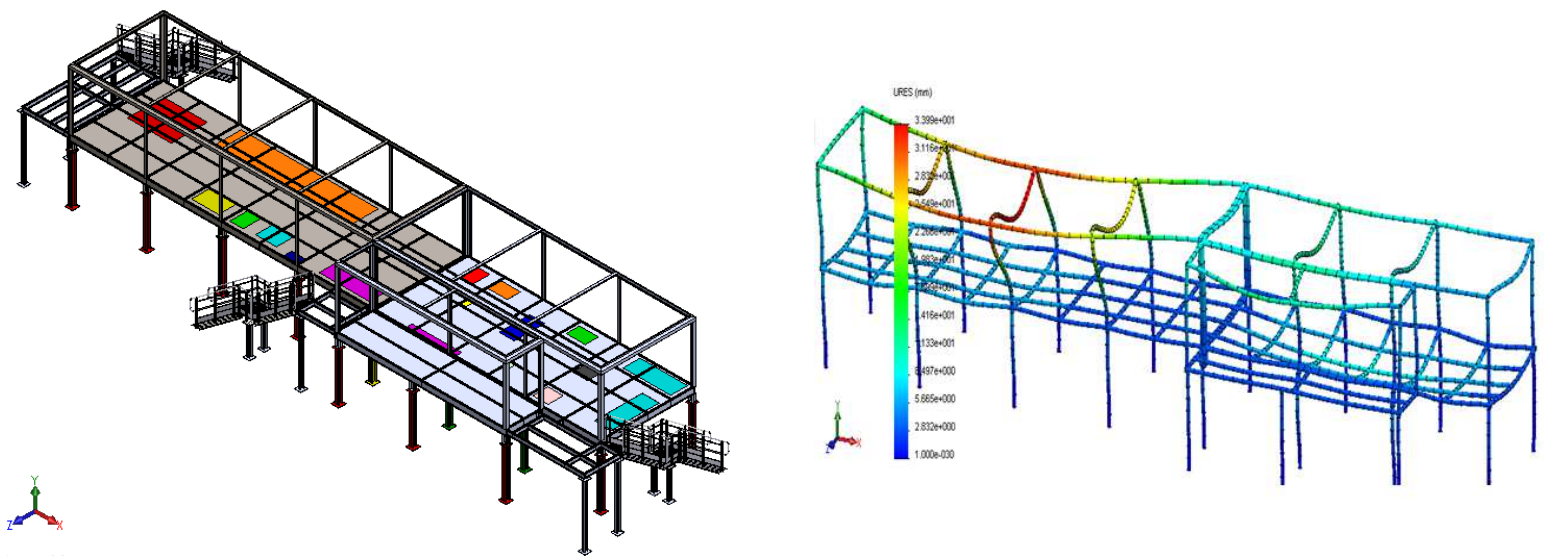

Figura 1: Exemplo de análise estrutural mecânica de eletrocentro

A construção do eletrocentro é formada principalmente por perfis estruturais de aço, chapas de aço e painéis para isolamento térmico. $O$ conjunto deve ser projetado para alcançar um grau de proteção adequado a instalação, bem como possuir sistemas específicos para pressurização e climatização internas da subestação.
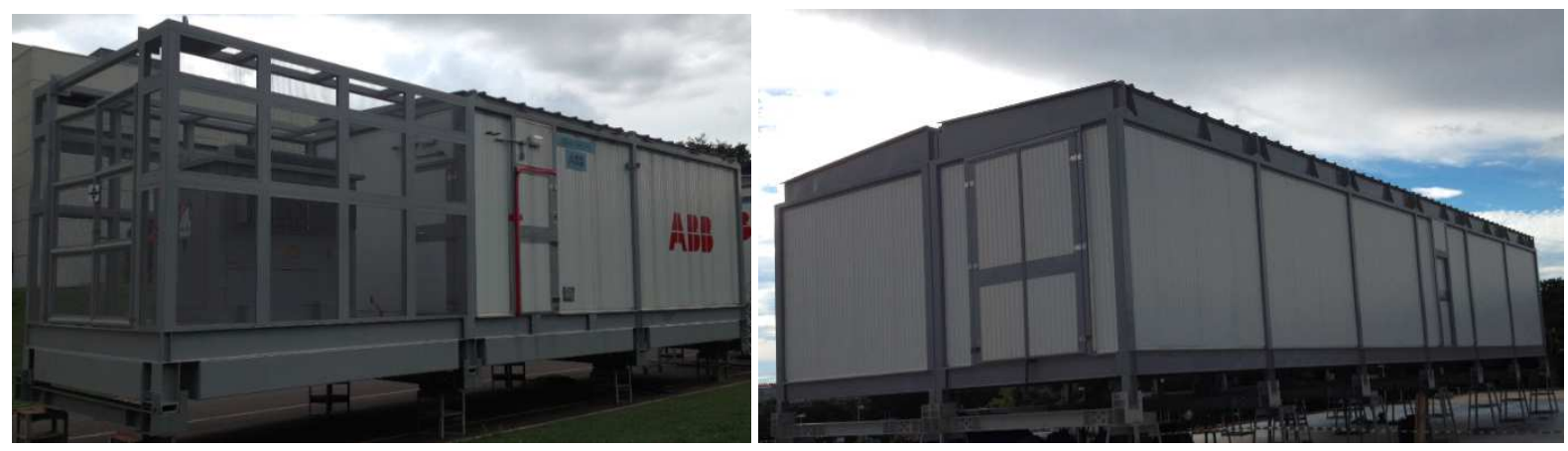

Figura 2: Conjunto mecânico fabricado

Além dos módulos principais que compôem o eletrocentro fazem parte do conjunto completo da subestação elétrica tipo eletrocentro: ante-câmaras, escadas, plataformas de pessoas e equipamentos, pilotis ou colunas de sustentação dos 
módulos e até as baias de transformadores que são fornecidas em estruturas metálicas.

\subsection{Infra-Estruturas e Sistemas Eletromecânicos dos Eletrocentros}

Como toda subestação elétrica, os eletrocentros são fabricados com toda a infraestrutura necessária para instalação dos painéis elétricos e de automação necessários para o funcionamento. Essa infra-estrutura contempla:

- Bandejamento e Cabeamento Internos que são instalados no porão de cabos do eletrocentro, sendo que são respeitados a separação por níveis de tensão entre cabeamento de potência, controle e comunicação;

- Aterramento da estrutura, bandejas e painéis com a disponibilidade de barras de equipotencialização no eletrocentro;

- lluminação e tomadas com a disponibilidade de paineis auxiliares de distribuição das mesmas;

- Sistema de Proteção contra Descargas Atmosféricas (SPDA) que para a solução mecânica do eletrocentro torna a própria estrutura metálica como captor natural, eliminando a necessidade de um sistema dedicado;

- Sistema de Detecção, Alarme e Combate à Incêndio (SDACI) utilizando um sistema de controle central a ser integrado em uma única supervisora na Brigada de Incêndio, sendo que a definição do agente de combate segue as especificações do cliente (Novec, Aerosol etc);

- Sistema de Pressurização e Climatização (HVAC) da subestação elétrica para controles de pressão e temperatura nominais a operação. A solução normalmente utilizada para eletrocentros é evaporadora do tipo precisão com sistemas de dutos de distribuição de ar climatizado. Também se utiliza para Skids sistemas menores do tipo wall-mounted;

- Sistema de Controle de Acesso afim de garantir segurança nas supervisões e operações da subestação localmente. Esse sistema é interligado diretamente a um servidor com cadastros dos funcionários habilitados para as operações;

- Rotas de Fuga com sinalização de emergência para correta evacuação de pessoas da sala em caso de necessidades de segurança.
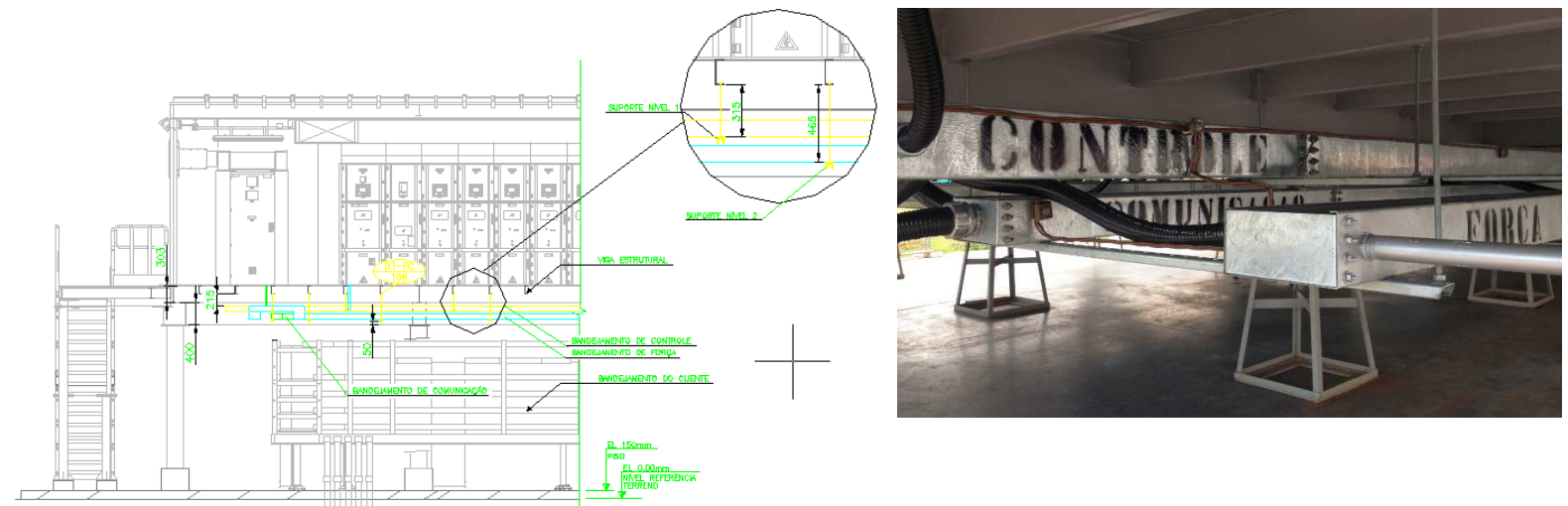

Figura 3: Níveis de bandejamento no porão de cabos do eletrocentro

O projeto de instalação dessa infra-estrutura e sistemas eletromecânicos são considerados para que todas as etapas de conexão em fábrica e reconexão em campo sejam realizadas com rapidez em virtude da modularização dos 
eletrocentros. Para que isso ocorra existem detalhes de fabricação como a utilização de diferentes tipos de caixas de junção entre módulos para facilitar a reconexão dos diversos tipos de cabos que compõem os sitemas de utilidades da subestação. Similarmente todo o projeto de bandejamento e dutos de ar condicionado são concedidos para facilitar a segregação dos módulos após montagem e testes em fábrica e envio para campo.
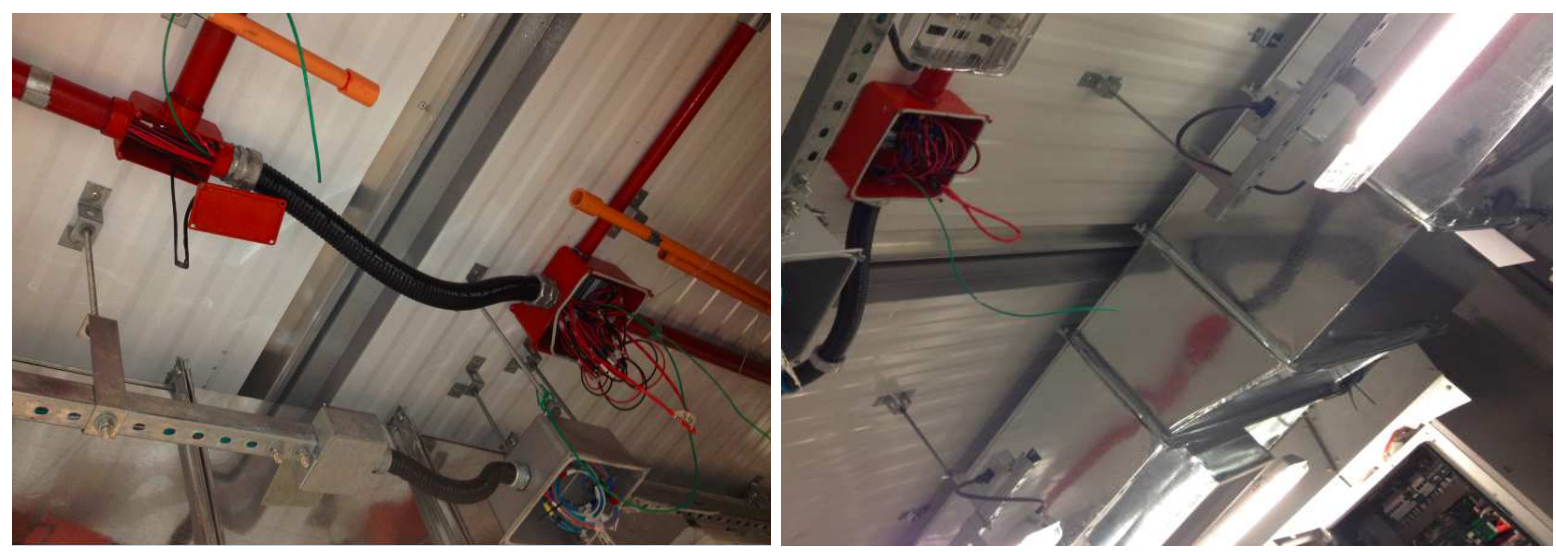

Figura 4: Facilidades de conexões e reconexões da infra-estrutura do eletrocentro

\subsection{Equipamentos dos Sistemas de Eletrificação e Automação das Subestações}

Os equipamentos do sistema de eletrificação que compõem a subestação elétrica do tipo secundária são reponsáveis pela distribuição e controle de cargas de média e baixa tensão em uma planta industrial.

Os equipamentos elétricos são separados por níveis de tensão e funções sendo que para uma subestação secundária podem ser exemplicados:

- Cubículos de distribuição de 34,5 kV do tipo Gas Insulated Switchgear (GIS);

- Cubículos de distribuição e centro de controle de motores (CCM) de 13,8 ou 4,16 kV do tipo Air Insulated Switchgear (AIS);

- Painéis de distribuição e centro de controle de motores (CCM) de 0,48 kV;

- Inversores de frequência para motores em média e baixa tensão;

- Transformadores de potência do tipo seco ou a óleo dependendo da aplicação.

Adicionalmente aos principais equipamentos do sistema de eletrificação, fazem parte do conjunto auxiliares importantes para a operação da subestação elétrica:

- Filtros de harmônicos e bancos de capacitores;

- Resistores de aterramento;

- No-breaks, retificadores e bancos de baterias;

- Geradores de emergência;

- Painéis auxiliares de distribuição.

É importante destacar dentro da solução de eletrificação a disponibilidade dos chamados relés inteligentes para acionamentos de motores com protolocos de comunicação do tipo Profibus ou Profinet e também dos dispositivos eletrônicos inteligentes (IED's) com protocolos de comunicação baseada na norma IEC 61850 permitindo uma integração elétrica com o sistema de automação de processos em uma arquitetura única, além da redução significativa do tradicional cabeamento de 
controle entre cubículos para intertravamentos internos e também para monitoração e comando via hardwired com os painéis de automação.

Essa arquitertura integrada propõe um único sistema integrado para a automação de processo e automação elétrica, permitindo a utilização dos mesmos componentes de hardware e software dentro de único sistema de automação. Por exemplo, os típicos controladores de processo podem ser utilizados para aplicações de processo como em malhas de controle (PID), mas também para funções de gerenciamento de energia como rejeição/limitação de cargas e sincronismo. Essa plataforma de integração, combinando sistema de controle de processo, sistema de controle elétrico, históricos de dados e monitoramento de ativos, permite a melhor tomada de decisões para o gerenciamento da produção, energia e manutenção dos ativos da planta.

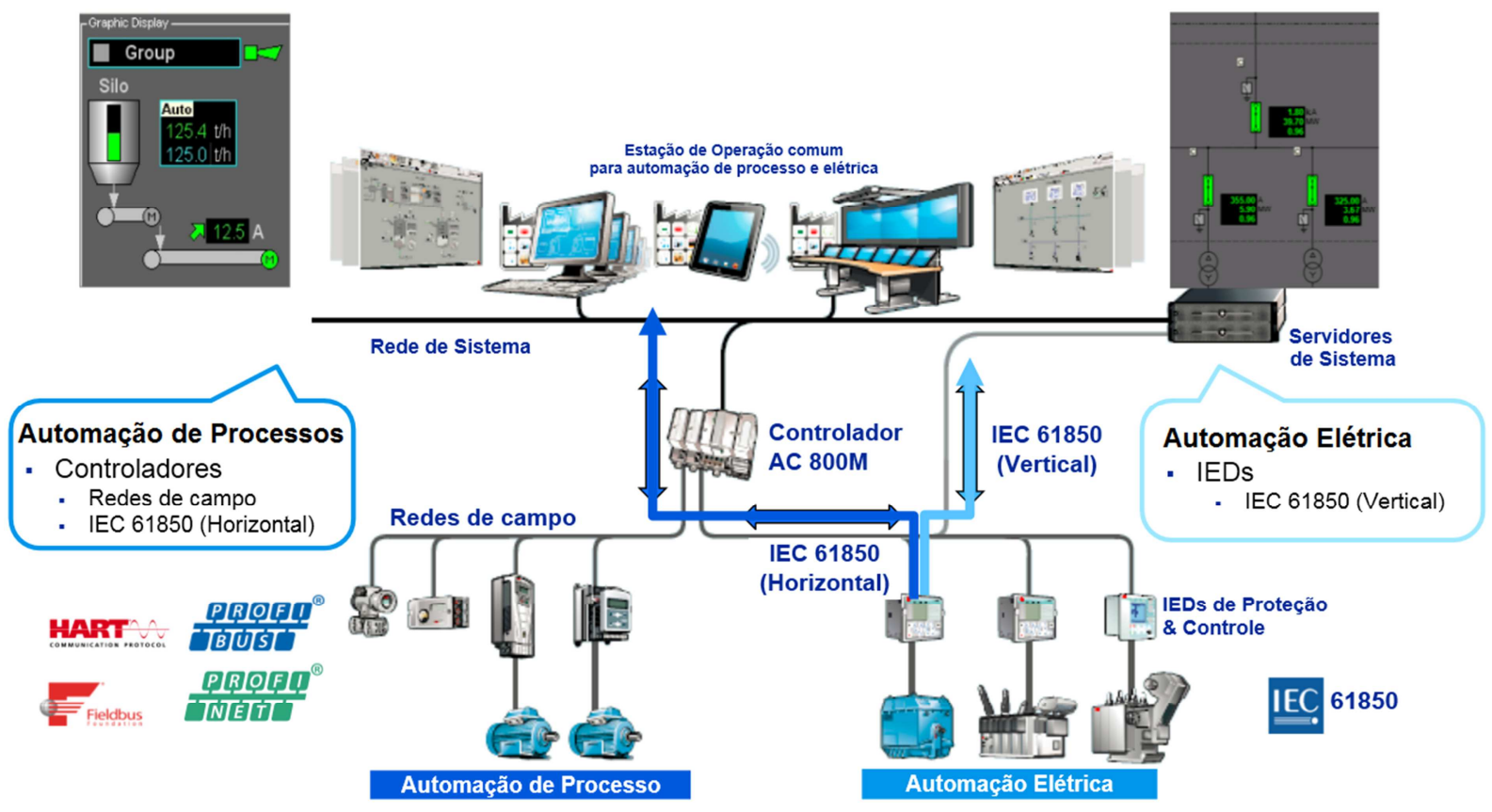

Figura 5: Arquitetura integrada de automação de processo e elétrica

Os equipamentos comuns dessa arquitetura integrada de automação dentro das subestações são:

- Painéis de controladores de processo;

- Painéis de ativos de comunicação como switches Ethernet;

- Painéis com cartões de entradas e saídas físicas (digitais e analógicas);

- Painéis de Relés de Segurança;

- Painéis de interface homem-máquina (IHM) ou estações de operação locais.

\section{SOLUÇÕES DE ELETROCENTROS E SISTEMA INTEGRADO DE AUTOMAÇÃO}

O conceito de sistema integrado de automação traz diversas vantagens a solução dos eletrocentros durante as fases de montagem e testes tanto em fábrica como em campo.

A redução significativa do cabeamento de controle entre cúbículos elétricos e também entre os painéis de entradas e saídas hardwired do tradicional sistema de automação possibilita uma maior rapidez durante o período de lançamento e 
interconexões de tais cabos em fábrica. Estima-se uma redução em torno de $50 \%$ de cabos e conexões dentro de uma subestação que não se limitaria somente a cabos mas todo o material associado a essas conexões e principalmente mão-de-obra para execução. Esse benefício é também transferido para a etapa de reconexão em campo cujos tempos de comissionamento são reduzidos.

Outro importante avanço com a utilização e padronização da solução de IED's e relés de proteção inteligentes se refere a pré-definição durante a fase de projeto de típicos lógicos para o correto funcionamento dos disjuntores e acionamentos. Esses típicos lógicos são facilmente replicados nos IED's e relés inteligentes através do carregamento do software aplicativo dos mesmos e reduz drasticamente o tempo de testes em fábrica e em campo, além de manter uma padronização de programação para a futura equipe de manutenção da planta. Adicionalmente aos típicos lógicos é fundamental para o teste funcional completo da subestação que todos os estudos de coordenação e seletividade elétricas dos equipamentos estejam disponíveis para a correta parametrização de proteção dos IED's e relés inteligentes.

É através do sistema integrado que o teste funcional da subestação considera não somente o teste local de determinado cubículo elétrico mas sim o conjunto do sistema elétrico com o sistema de automação validando os testes funcionais remotos, registros de alarmes e eventos, e monitoramento dos ativos. Assim, somente em um ambiente de fábrica e com a disponibilidade e instalação de todos os componentes da subestação elétrica se conseguiria uma eficiência significativa nesses testes integrados e é com esse argumento central que a solução de eletrocentro se encaixa no contexto possibilitando a disponibilidade completa da subestação elétrica para testes em fábrica.

Os benefícios dessas soluções integradas não devem se limitar somente a economia no CAPEX (capital expenditure) durante as fase de fabricação e implantação como expostos nos pontos acima e também nas reduções dos custos de construção civil e montagem em campo das tradicionais subestações elétricas de alvenaria, mas a maior margem se concentra no OPEX (operational expenditure) em virtude das diversas oportunidades de melhorias na manutenção, segurança e operação da planta industrial como um todo. Do ponto de vista de manutenção, normalmente os ativos dos sistemas elétricos não são tratados com tanta importância quanto os grandes equipamentos mecânicos que compõem uma planta industrial e suas ações de manutenção se limitam de maneira corretivas pela falta de informações para se ter o correto algoritmo de monitoramento de tais ativos elétricos. Uma arquitetura integrada eleva a possibilidade de padrões Ethernet como a IEC 61850 e a Profinet para o monitoramento da saúde vital dos equipamentos da planta. A cadeia completa dos ativos de um determinado equipamento mecânico desde os seus relés de proteção na subestação elétrica até transmissores de vibração e temperatura no campo podem ser facilmente visualizadas dentro do contexto de sistema integrado de processo e elétrica permitindo um refinamento do algoritmo de monitoração e consequentemente das estratégias de manutenção preditiva do ativo como um todo afim de se minimizar os tempos de parada e reduções de produção. A integração com sistemas de gerenciamento de manutenção (CMMS) também é uma oportunidade amplificada nessa topologia.

Explorando um exemplo prático de ativo do sistema elétrico como um típico disjuntor, aonde sua saúde é passível de monitoramento através de uma variedade de dados e parâmetros disponibilizados nos atuais IED's para definição da estratégia de manutenção, seguem alguns desses parâmetros:

- Operation Monitoring Time: tempo de inatividade do disjuntor; 
- Breaker Contact travel Time: tempo de abertura e fechamento do disjuntor;

- Operations Cycle Counter: contator de operações sobre o disjuntor;

- Spring Charge Indication Time: tempo de carregamento de mola do disjuntor;

- Breaker Wear: vida útil do disjuntor em função da quantidade de trips e correntes submetidas;

- Gas Pressure Supervision: monitoramente de pressão em disjuntores isolados a gás $\left(S F_{6}\right)$.

O sistema integrado de automação também siginifica integração entre operações de processo e energia na planta principalmente no contexto de sala de controle central (SCC) aonde engenheiros e operadores de processo, engenheiros elétricos e equipes de manutenção trabalham juntos em um mesmo ambiente coloborativo permitindo uma melhor tomada de decisões uma vez que possuem acessos a todas as informações pertinentes para cada disciplina através de comunicações remotas com as subestações elétricas e instrumentações dos equipamentos da planta, além dos monitores de ativos e produção da planta.

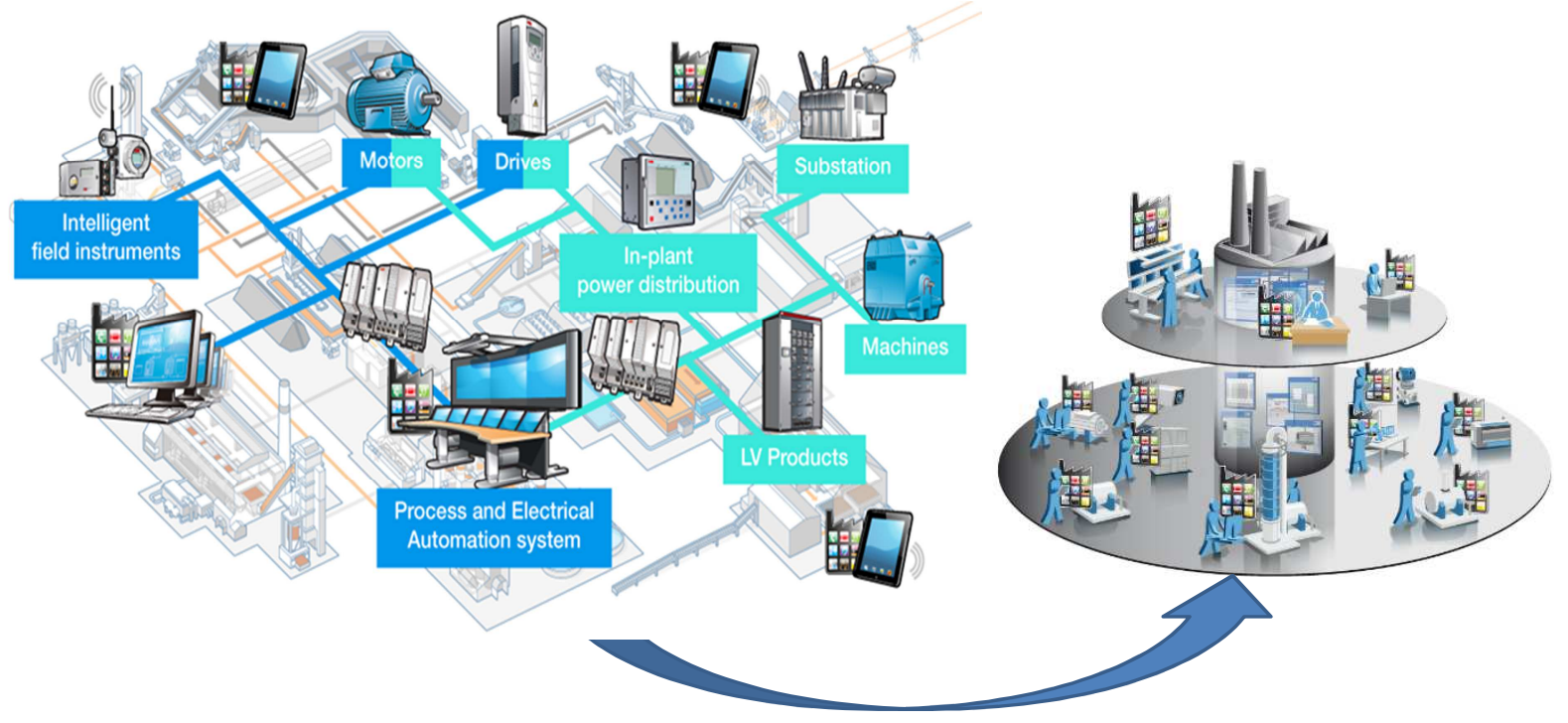

Figura 6: Ambiente colaborativo entre operações da planta através do conceito de SCC

\section{CONCLUSÃO}

As vantagens da solução de eletrocentros visando melhor gerenciamento na implantação de plantas industriais com reduções de tempos de montagem e comissionamento em campo são apenas parte dos benefícios dessa solução pois abrande somente o CAPEX.

Do ponto de vista de OPEX, a associação da solução de eletrocentros com o conceito de sistema integrado de automação entre processo e elétrica permite a abertura de diversas oportunidades de melhorias operacionais através da disponibilidade de diferentes informações em um mesmo ambiente colaborativo de tomada decisões dentro de uma sala de controle central. Adicionalmente, a implantação de um sistema de manunteção preditiva na planta poderá ser mais eficiente devido a maior quantidade de parâmetros e informações dos ativos dos sistemas elétrico e de processo disponibilizados, possibilitando uma melhor definição das estratégias de manutenção dos equipamentos da planta. 
Assim, apesar de soluções distintas, eletrocentros e sistema integrado de automação são avanços tecnológicos cujos ganhos de implantação e operação tendem a ser amplificados quando planejadas em conjunto.

\section{BIBLIOGRAFIA}

1 Vasel, Jeffrey. One Plant, One System: Benefits of Integrating Process and Power Automation. Texas A\&M Relay Conference, 2012.

2 Monaco, Leandro. O Futuro do Gerenciamento de Planta Industrial: Um Enfoque sobre a Energia - Estudo de Caso Vale S11D. Brazil Automation 2014, 2014. 